\title{
MIMO Detection and Equalization for Single Carrier Systems using the Alternating Direction Method of Multipliers
}

\author{
Nuno Souto, Member, IEEE, and Rui Dinis, Member, IEEE
}

\begin{abstract}
In this letter, a multiple-input multiple-output (MIMO) detection algorithm based on the alternating direction method of the multipliers (ADMM) is proposed for single carrier transmissions in time dispersive channels. The ADMM is applied as a heuristic to solve the maximum likelihood detection problem for arbitrary signal constellations formulated in the frequency domain. This approach allows most of the decoding steps to be implemented in the frequency domain with an overall reduced complexity cost. Simulation results show that performances close to the matched filter bound can be attained in large problem sizes, even in overloaded scenarios.
\end{abstract}

Index Terms - Nonconvex optimization, ADMM, MIMO detection, SC-FDE.

\section{INTRODUCTION}

The high spectral efficiency achievable with multiple-input multiple-output (MIMO) systems has attracted enormous research activities, with the joint detection of multiple transmitted symbols playing a central role in order to grasp the theoretical gains [1].

The non-deterministic polynomial time-hard (NP-hard) nature of the MIMO detection problem [2] has led to the development of numerous sub-optimal MIMO detectors, especially for large problem sizes. These detectors are often studied assuming memoryless channels which may be adequate for single carrier (SC) transmission in narrow-band channels [3] or orthogonal frequency-division multiplexing (OFDM) systems in frequency-selective channels [4]. Due to its lower envelope fluctuations, SC transmission is an attractive alternative to OFDM for the uplink of broadband wireless systems and it has been adopted for the long term evolution (LTE) standards [5]. However, for broadband systems, the multipath channel often becomes severely time dispersive leading to high intersymbol interference (ISI) levels. Therefore, in SC-MIMO systems, the receiver should be able to cope with this induced ISI. Adopting a zero-padded or a cyclic prefix (CP) assisted block transmission structure eliminates inter-block interference which reduces the problem

$\mathrm{N}$. Souto is with the ISCTE-University Institute of Lisbon and Instituto de Telecomunicações, 1649-026 Lisboa, Portugal (e-mail: nuno.souto@1x.it.pt).

R. Dinis is with the FCT - Universidade Nova de Lisboa and Instituto de Telecomunicações, 2829-516 Caparica, Portugal (e-mail: rdinis@fct.unl.pt).

This work was partially supported by the FCT - Fundação para a Ciência e Tecnologia and Instituto de Telecomunicações under project UID/EEA/50008/2013. of error propagation and allows the use of flat fading based MIMO detectors at the block level. Even though the special structure of the channel matrix can be exploited [6], the combination of block size, multiple antennas, high order modulations and long channel memory can result in a very large problem size making the use of frequency domain processing an often more appealing approach [7], [8]. While frequency domain linear equalizers such as zero-forcing (ZF) and minimum mean squared error (MMSE) can be implemented with substantial lower complexity they usually perform quite far from the matched filter bound (MFB). Therefore, nonlinear schemes such as hybrid time-frequency decision feedback equalizers (DFE) [7], [9], [10], are often preferred, with the receivers based on the iterative block decision feedback (IB-DFE) equalizer [8], [9] being the most promising ones due to their excellent performances. Still, the matrix inversions required for each frequency and iteration of the IB-DFE can lead to significant receiver complexity which is particularly relevant in large problem settings. A different approach was employed in [11] using message passing on graphical models. The proposed algorithms can achieve near optimal performance for large dimensions but the complexity order is dependent on the block size and limited to BPSK/QPSK. There are also other alternative approaches especially designed for coded systems which adopt a turbo equalization strategy [9][12], however in this letter we focus on uncoded systems.

The alternating direction method of the multipliers (ADMM) is a widely used approach for solving convex optimization problems, for which it is guaranteed to converge under mild conditions, having numerous applications [13][14]. Although it was originally developed for convex problems, it has been shown recently that ADMM can also be used as a powerful heuristic for solving several nonconvex problems [14]-[19], regardless of the lack of theoretical convergence guarantees. Motivated by these results, in this letter we devise an algorithm based on the ADMM for the detection and equalization of SC transmissions over time dispersive MIMO channels, which is also a nonconvex problem. The algorithm is developed for arbitrary signal constellations, and takes advantage of the capability of ADMM to decompose the detection problem into a series of simpler subproblems with closed form solutions. Most of the processing steps are derived in the frequency domain in order to reduce the overall 